\title{
Interaction of Ising-Bloch fronts with Dirichlet boundaries
}

\author{
A. Yadav ${ }^{*}$ and D. A. Browne \\ Department of Physics and Astronomy, Louisiana State University, Baton Rouge, LA 70803-4001
}

\begin{abstract}
We study the Ising-Bloch bifurcation in two systems, the Complex Ginzburg Landau equation (CGLE) and a FitzHugh Nagumo (FN) model in the presence of spatial inhomogeneity introduced by Dirichlet boundary conditions. It is seen that the interaction of fronts with boundaries is similar in both systems, establishing the generality of the Ising-Bloch bifurcation. We derive reduced dynamical equations for the FN model that explain front dynamics close to the boundary. We find that front dynamics in a highly non-adiabatic (slow front) limit is controlled by fixed points of the reduced dynamical equations, that occur close to the boundary.
\end{abstract}

PACS numbers: 82.40.-g,05.70.Ln

\section{INTRODUCTION}

In spatially extended reaction-diffusion systems far from equilibrium, the interplay of the diffusion and reaction processes is frequently associated with the formation of spatial or temporal patterns in the concentration fields [1, 2, 3, 4, 5, 6]. One such example is a front-like structure connecting two different homogeneous steady states. In a bistable system, where both steady states are stable against small perturbations, these fronts can undergo bifurcations, known as nonequilibrium Ising-Bloch bifurcations, where a stationary Ising front exchanges stability with a pair of counterpropagating Bloch fronts. This bifurcation has been observed in several chemical reactions [4, 5, 6] and also in liquid crystals [7, 8, 9] subject to an external time-dependent perturbation.

Two models of this Ising-Bloch bifurcation have been extensively studied in this context. One is the parametrically forced Complex Ginzburg Landau equation (CGLE) [7]. This system describes nematic liquid crystals subjected to a rotating magnetic field and a high frequency electrical field [8]. The CGLE is often used in spatially extended systems to describe the dynamics close to an oscillatory instability (Hopf bifurcation). The other is a FitzHugh Nagumo (FN) model [4, 10, 11, 12, 13], which qualitatively models various chemical reactions 14, 15, 16]. Front solutions in this model have been extensively investigated, specially when translational invariance is broken by the presence of spatial inhomogeneities, which is often the case in realistic experimental situations. This includes the ways in which one Bloch front can be perturbatively changed to the other (leading to front reversal) 10, 11, 12, 13]. Particularly, one such scenario for front reversals and other exotic nonuniform (variable velocity) front motion like breathing, involves the breaking of translational invariance by zero flux boundary conditions [4]. This nonuniform motion of fronts is explained by the presence of uniform velocity front solutions (nullclines of the FN model

*Electronic address: yadav@phys.lsu.edu partial differential equations) to which faster or slower moving fronts relax adiabatically.

In this paper we examine nonuniform front motion in the case when translational invariance is broken by imposing fixed chemical concentrations at the boundary of a reactor (Dirichlet boundary conditions). An adiabatic description of nonuniform front motion along the lines of [4] is inadequate. It relies on nullclines and fails to explain the jumps from one nullcline to another or the influence of fixed points. Therefore, we employ a dynamical approach which satisfactorily accounts for jumps and fixed point influence. We also establish the generic nature of nonuniform front motion in the presence of spatial inhomogeneities by studying it in two distinct systems, the FN and CGL models.

The interaction of traveling fronts with boundaries for Dirichlet boundary conditions shows several new features. We see a transition from front reversal to trapping of an incoming Bloch front on its approach to a boundary as a function of boundary values. We also find that trapped fronts and reversed fronts can coexist for certain boundary conditions. Finally, we derive reduced dynamical equations that explain the features mentioned above.

In section II of this paper we review the relevant details of the CGL and FN models. Section III presents our numerical study of the two models. Section IV contains an analytic study of front interactions with boundaries for the FN model. Our conclusions are listed in Section $\mathrm{V}$.

\section{THE MODELS}

The parametrically forced CGLE is the generic model describing the slow phase and amplitude modulations of a spatially distributed assembly of coupled oscillators near its Hopf bifurcation [17]. This assembly of autooscillators is parametrically forced at twice its natural frequency and can be written as,

$$
\begin{aligned}
\frac{\partial A}{\partial t}= & (\mu+i \nu) A+(1+i \alpha) \nabla^{2} A \\
& -(1+i \beta)|A|^{2} A+\gamma A^{*}+\kappa .
\end{aligned}
$$


The complex field $A$ contains the amplitude and phase of the coupled oscillators, $\mu$ measures the distance from the oscillatory instability threshold, $\nu$ is the detuning of the forcing term frequency from exactly twice the Hopf frequency, $\alpha$ and $\beta$ are real control parameters, and $\gamma>0$ is the forcing amplitude at twice the natural frequency. The right hand side of Eq. (11) cannot be written in a variational form if $\nu, \alpha$, and $\beta$ are nonzero.

The parameter $\kappa$ represents parametric forcing at the natural frequency of the system. If $\kappa=0$, Eq. (11) has a the parity symmetry $A \rightarrow-A$, and the nonequilibrium Ising-Bloch bifurcation is a symmetric pitchfork [7, 18] with the front velocity as the order parameter. Zero velocity Ising walls lose stability to counterpropagating Bloch walls, as the bifurcation parameter $\gamma$ crosses its critical value. The pitchfork unfolds into a saddle node bifurcation for a nonzero $\kappa$ where along with the stable Ising wall, a stable and unstable pair of Bloch walls appear at the bifurcation.

The CGLE Eq. (1) has trivial homogeneous solutions $(A(x)= \pm \sqrt{\mu+\gamma}+0 i)$ in the variational case $(\alpha=\beta=$ $\nu=0)$ making it bistable. Solutions connecting these trivial states are,

$$
A(x)=\sqrt{\mu+\gamma} \tanh \left(\sqrt{\frac{\mu+\gamma}{2}} x\right)+0 i,
$$

which is the Ising wall solution characterized by a zero imaginary part, and

$$
A(x)=\sqrt{\mu+\gamma} \tanh (\sqrt{2 \gamma} x)+i \sigma \sqrt{\mu-3 \gamma} \operatorname{sech}(\sqrt{2 \gamma} x),
$$

the two Bloch wall solutions distinguished by their respective chirality $\sigma= \pm 1$. In the case when nonvariational parameters $(\alpha, \beta, \nu)$ are non-zero, and $\gamma^{2}>$ $(\nu-\beta \mu)^{2} /\left(1+\beta^{2}\right)$, Eq. (1) is still bistable and has trivial solutions,

$$
\begin{aligned}
A & =R \exp (i \phi) \\
R^{2} & =\frac{\mu+\beta \nu+\left[\gamma^{2}\left(1+\beta^{2}\right)-(\nu-\beta \mu)^{2}\right]^{1 / 2}}{\left(1+\beta^{2}\right)} \\
\cos (2 \phi) & =\left(-\mu+R^{2}\right) / \gamma \\
\sin (2 \phi) & =\left(\nu-\beta R^{2}\right) / \gamma .
\end{aligned}
$$

Nonvariational Ising and Bloch wall solutions are qualitatively different from their variational counterparts. Nonvariational Bloch walls move as a result of the breaking of chiral symmetry [7], whereas variational Bloch walls are stationary. Chirality breaking is unlike other mechanisms of front motion, where a globally stable state invades an unstable or metastable state [19, 20]. In the perturbative limit when $(\alpha, \beta, \nu)$ are small, the velocity of Bloch fronts can be written as

$$
c=\chi\left[\frac{\mu+\gamma}{2 \gamma}\right]^{\frac{1}{2}} \frac{3 \pi}{2(3 \mu-\gamma)}[-\nu+\beta \mu+(\alpha-\beta) \gamma],
$$

where $\chi= \pm \sqrt{3\left(\gamma_{c}-\gamma\right)}$. The Bloch wall velocity $c$ is proportional to $\sqrt{\gamma_{c}-\gamma}$ as expected for a pitchfork bifurcation.
Now we look at the FN model, which also shows a front bifurcation. This is a simple two component model and has been thoroughly analysed in Ref. 10, 11, 12, 13, 21 in the context of this bifurcation. It has been widely used to model patterns in reactions like the Belousov-Zhabotinsky (BZ) reaction 22, 23, 24, 25, 26], Ferrocyanide-Iodate-Sulfite (FIS) reaction 44 and Chlorite-Iodide-Malonic-Acid reaction(CIMA) 14, 15, 16. The two component reaction-diffusion system, with $v(x, t)$ impeding the production of $u(x, t)$, is given by

$$
\begin{aligned}
& \frac{\partial u}{\partial t}=\epsilon^{-1}\left(u-u^{3}-v\right)+\delta^{-1} u_{x x} \\
& \frac{\partial v}{\partial t}=\left(u-a_{1} v-a_{o}\right)+v_{x x} .
\end{aligned}
$$

The parameters $\epsilon$ and $\delta$ differentiate the time scales and space scales of the two fields respectively. The parameters $a_{1}$ and $a_{o}$ characterize renormalized local reaction parameters, possibly after an adiabatic elimination of faster reacting species. Equations (4) are in general nonvariational except for certain specific parameter values. The parameter $a_{o}$ is analogous to the parameter $\kappa$ in the CGL equation, and it controls the symmetry of the front bifurcation. If $a_{o}=0$, the FN model undergoes a symmetric front bifurcation represented by a pitchfork. For a nonzero $a_{o}$ the pitchfork unfolds into a saddle node as in the CGLE. A notable difference between the two systems is the presence of the parameter $\delta$ in the FN model. This parameter affects the relative spatial extent of the fronts connecting the trivial homogeneous solutions of Eq. (4). Thus, by choosing a suitable $\epsilon / \delta$ ratio, the connecting fronts of one of the fields can be made very sharp compared to the other. This is not possible in the CGLE, where fronts for both the real and imaginary parts have the same spatial extent.

Trivial homogeneous solutions to the FN model are

$$
u_{c}= \pm \sqrt{\frac{a_{1}-1}{a_{1}}} \quad v_{c}=\frac{u_{c}}{a_{1}} .
$$

Similar to the CGLE, the FN model has Ising and Bloch fronts as its solutions, that bifurcate in a pitchfork. The bifurcation parameter in the FN model may be chosen to be $\eta=\sqrt{\epsilon \delta}$. The critical value of this parameter is $\eta_{c}=(3 / \sqrt{(2) 2})\left(a_{1}+1 / 2\right)^{3 / 2}[12]$. The Bloch wall velocities given by

$$
c^{2}=\frac{6 \sqrt{2}}{\eta_{c}^{2}\left(\sqrt{a_{1}+1 / 2}\right)}\left(\eta_{c}-\eta\right),
$$

are proportional to $\sqrt{\eta_{c}-\eta}$, the deviation of the bifurcation parameter from its critical value, as expected for a pitchfork bifurcation.

As discussed above, both these models have common features associated with the front bifurcation. This forms the basis of their comparative study in the forthcoming 
sections. Front dynamics in both these systems can be represented by the system of equations,

$$
\begin{aligned}
\dot{x} & =c \\
\dot{c} & =\left(\rho_{c}-\rho\right) c-g c^{3} .
\end{aligned}
$$

These equations employ the pitchfork bifurcation normal form with velocity as the order parameter, coupled with the trivial observation that the velocity is the rate of change of position. The front velocity $c$ and position $x$, therefore constitute two degrees of freedom, that are sufficient to describe front dynamics close to the front bifurcation. The bifurcation parameter $\rho$ is denoted by $\gamma$ for the CGL equation and $\eta_{c}$ for the FN model. The two independent variables $c$ and $x$ in Eq. (7), which are obviously uncoupled, represent dynamics where translational invariance is present, and the solutions are independent of a choice of spatial origin. Scenarios can be envisioned where this translational invariance is broken. Examples are the imposition of different boundary conditions [4] or spatial inhomogeneities introduced externally via an advective field [12]. To account for broken translational invariance in these scenarios, one has to modify Eq. (7). This would lead to the coupling of the two degrees of freedom $x$ and $c$, the details of which would depend on the scenario considered. In this paper, we introduce a spatial inhomogeneity in the form of Dirichlet boundary conditions, examining its effect on incoming Bloch fronts in both models, and drawing parallels. The next section constitutes our numerical study. In section IV, we derive and analyse the way in which Dirichlet boundary conditions couple $x$ and $c$ for the FN model. Such an analysis is not possible for the CGL equation since it is impossible to represent the front position by a single point. Therefore, we rely exclusively on numerical simulations for that equation.

\section{NUMERICAL RESULTS}

In this section we lay out the numerical details of the study of the CGL and FN models. The simulations in the two models are carried out in regimes where analytical calculations performed by reducing front dynamics to a fewer degrees of freedom are not possible. This is the regime where Bloch fronts have high velocities (far beyond the front bifurcation threshold), and the fields forming the front core, have similar spatial scales.

We solve both the CGL and FN system of reaction diffusion equations using an implicit Crank-Nicholson scheme. Dirichlet boundary conditions are used at both ends of the domain, which is typically composed of 400 grid points with a time step size of 0.01 . The boundary values at one end are fixed at one of the homogeneous solutions of the Eq. (11) and (4). At the other end we are free to vary the boundary condition. In our numerical simulations, we keep the domain large compared to the characteristic spatial extent of the front, so that the influence of the boundary is only felt when the front is close
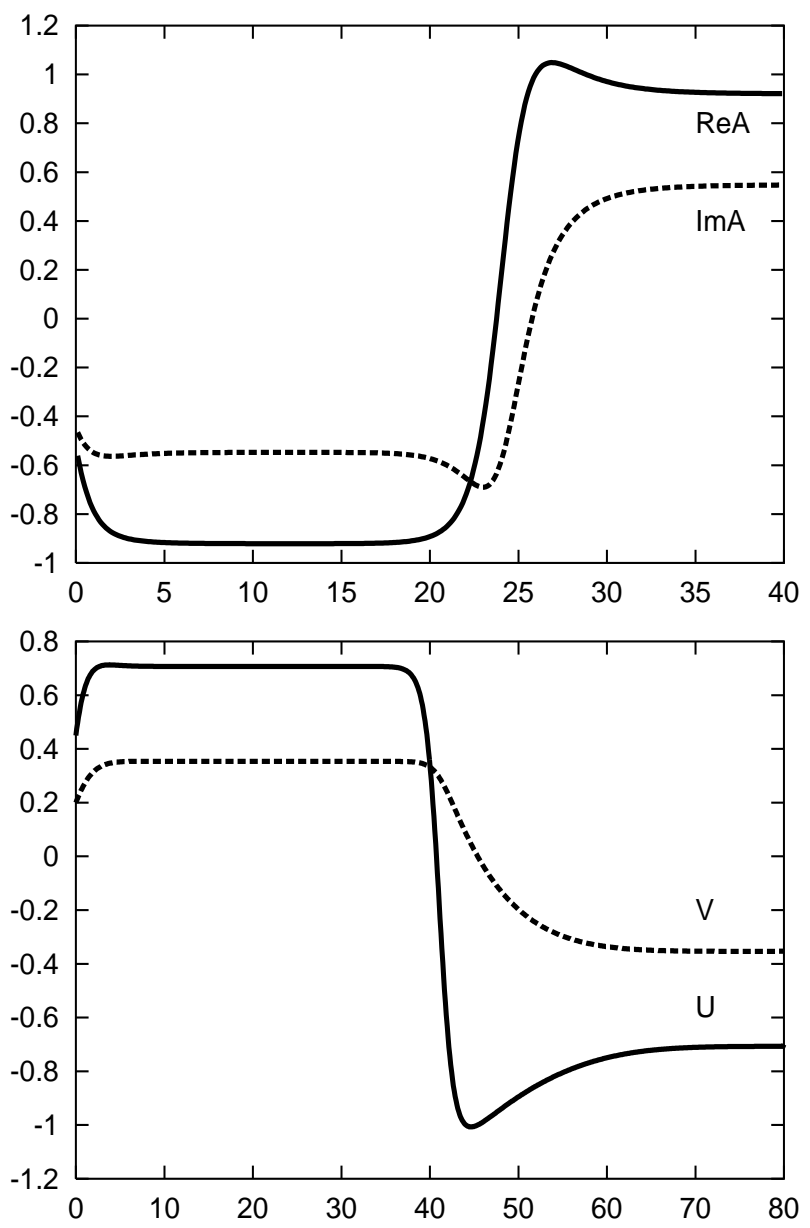

FIG. 1: (a) Typical traveling front for the CGLE (b) Traveling front in the FN model

enough to it. We verified that the grid and time steps were small enough to ensure that the numerical solution converged.

By a suitable choice of initial conditions a Bloch front or its counterpropagating partner can be generated. The symmetry of the bifurcation ensures that they have the same speed as long as they are not close to a boundary. A typical front for the parametrically forced CGL equation and the FN system of equations is shown in Fig. 10 Bloch fronts for the CGL model show a characteristic chirality broken structure at their core [] which is essential for their propagation. Similar structure considerations apply to fronts in the FN model [21].

In our simulations we focus on the interaction of incoming Bloch walls with boundaries, where Dirichlet boundary conditions are imposed on the two fields, $\operatorname{Re} A, \operatorname{Im} A$ in the CGL equation and $u, v$ in the FN model. Particularly we look at how the front cores are perturbed by the boundary for a whole range of boundary conditions. Fronts coming into the boundary from infinity in both models either rebound or get trapped depending on the Dirichlet boundary values. A front that traps loses 

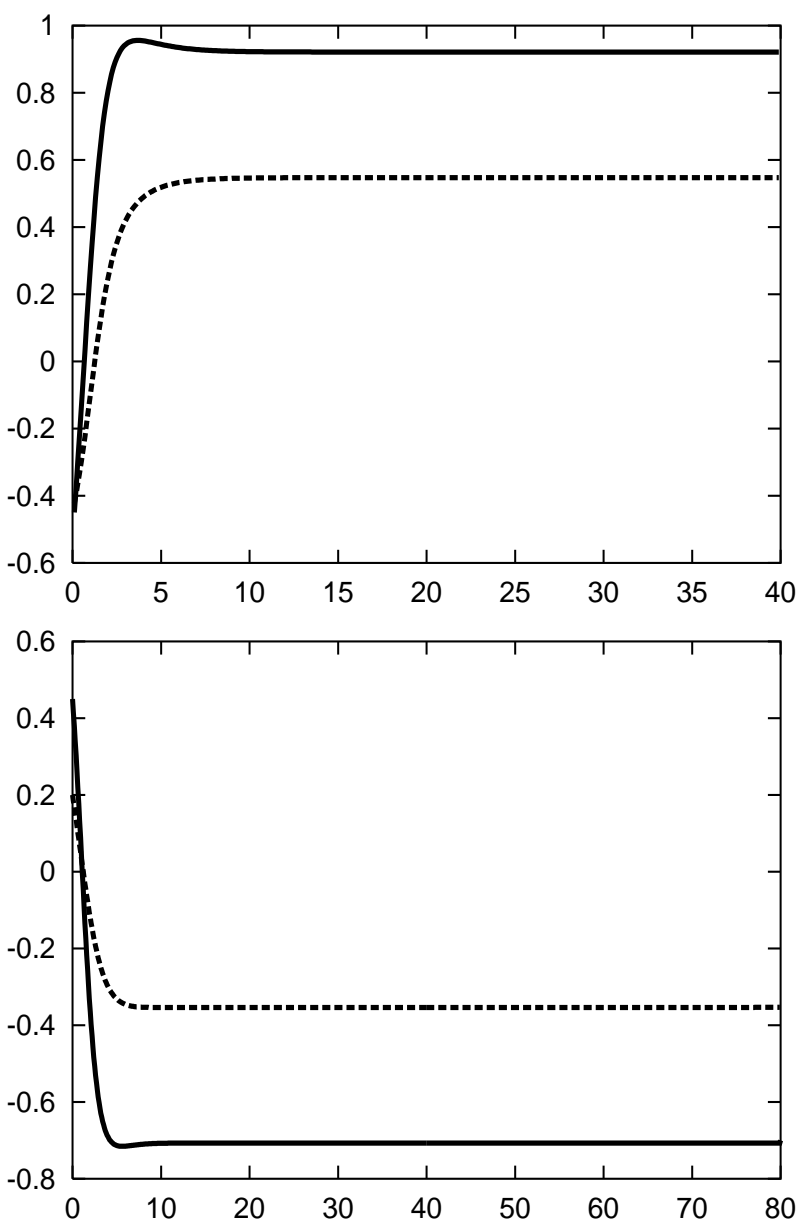

FIG. 2: (a) A typical Nontrivial stationary solution to which trapped fronts evolve into for the CGLE. The spatially homogeneous solutions are connected to the Dirichlet boundary values. (b) Nontrivial stationary solution for the FN model.

its core structure and evolves into the nearest available stable (attracting) configuration of the fields, which in this case is the nontrivial steady state solution of Eq. (II) or Eq. (4) for that particular boundary condition. Figure 2 shows a typical nontrivial steady state solution for the CGLE and FN model. This solution is comprised of a spatially homogeneous part, given by Eq. (2) for the CGLE or Eq. (5) for the FN model, and an inhomogeneous part that connects the spatially homogeneous solutions to the Dirichlet boundary value. Rebounding phenomena close to the boundary is characterized by the core of an incoming front flipping into the core of its counterpropagating partner, resulting in the front moving away.

Our observations are plotted in the plane of boundary values, revealing a curve separating regions of bouncing and trapped fronts for both CGL and FN systems. Figure 3 shows the transition curve for $\alpha=-0.1, \gamma=0.31$, $\beta=-0.15, \mu=1.0$ and $\nu=0.1$ for the parametrically forced CGL equation. A similar transition curve for the

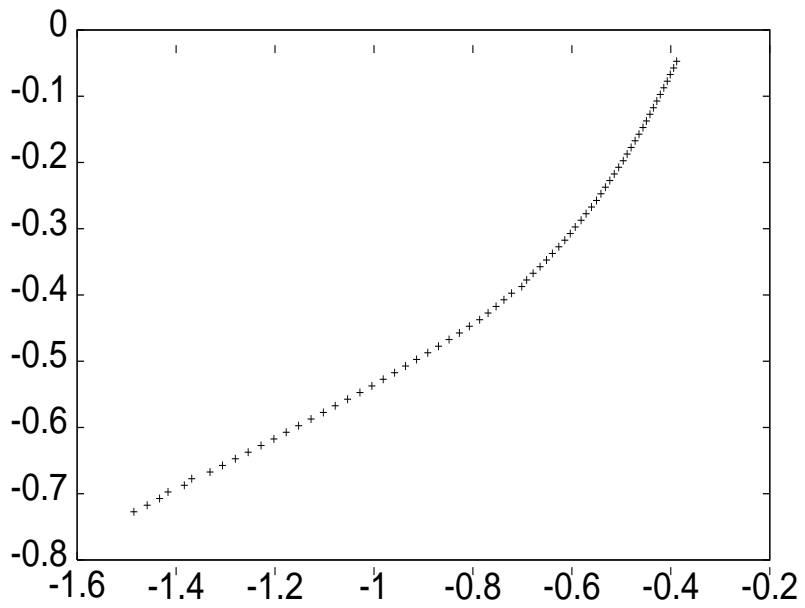

FIG. 3: Transition Curve from a region of trapped Bloch fronts to bouncing ones for the CGLE. ReA boundary value on the $\mathrm{X}$ axis, ImA boundary value on the $\mathrm{Y}$ axis

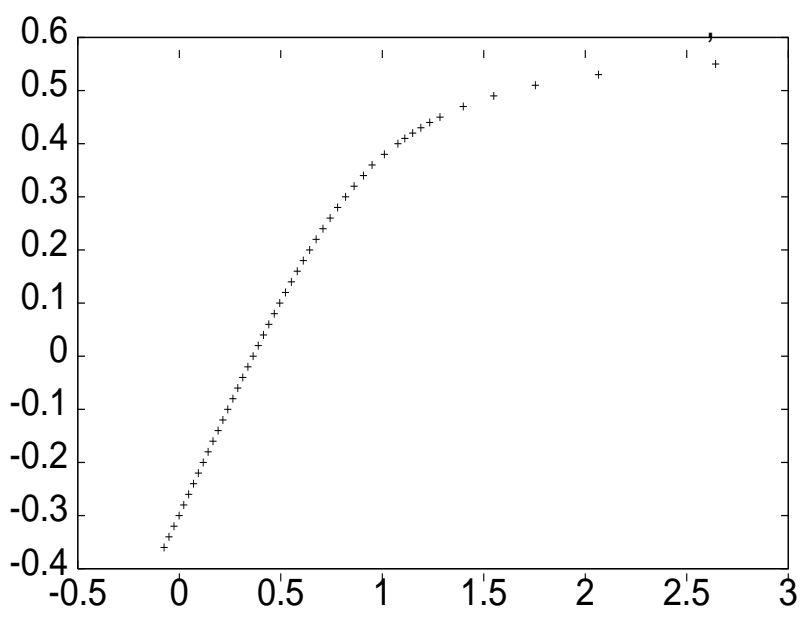

FIG. 4: Transition Curve from a region of trapped Bloch fronts to bouncing ones for the FN model. U boundary value on the $\mathrm{X}$ axis, $\mathrm{V}$ boundary value on the $\mathrm{Y}$ axis.

FN model, with $a_{1}=2.0, \delta=0.14, \epsilon=0.05$ is shown in Fig. 4. As one closes in on the curve from the trapping (bouncing) region, the fronts take longer to get trapped (bounce). This slowing down in the dynamics close to the transition curve is indicative of critical behavior, usually associated with eigenvalues of fixed points that approach zero when a parameter (the Dirichlet boundary value here) is varied. An analytical explanation of the slowing down is given in the next section.

Incoming Bloch fronts, in both the CGL and FN models, evolve into nontrivial stationary solutions to Eq. (1) and Eq. (4) respectively when trapped at the boundary. These nontrivial solutions are linearly stable by virtue of the Bloch fronts evolving into them. It remains to be ascertained whether these solutions remain stable when Dirichlet boundary values that lead to a bounce are imposed at the boundary. Hypothetically, one could as- 
sociate the loss of stability of these nontrivial solutions with the transition from trapping to bouncing of incoming fronts. We test this hypothesis by carrying out a numerical linear stability analysis of the nontrivial stationary solutions $\psi_{o}$. Eq. (1) is linearized to,

$$
\begin{aligned}
\frac{\partial \delta \psi}{\partial t}= & (1+i \alpha) \nabla^{2} \delta \psi+\left[\mu+i \nu-2(1+i \beta)\left|\psi_{o}\right|^{2}\right] \delta \psi \\
& +\left[\gamma-(1+i \beta) \psi_{o}^{2}\right] \delta \psi^{*}
\end{aligned}
$$

for the perturbation $\delta \psi=0$ at the boundaries. A similar linearization is done for the FN system. It is found that the eigenvalue spectrum for both systems has negative real parts in the trapping region, as expected. Moreover, these real parts remain negative when we evaluate the stability of the nontrivial stationary solutions in the bouncing region. Even though nontrivial solutions are stable in the bouncing region, incoming fronts do not evolve into them. Consequently, the critical behavior is not governed by the loss of stability of these solutions.

We also observe that close to the transition curve bouncing and trapped fronts can coexist. Instead of coming in from infinity and rebounding, a Bloch front created close to the boundary may get trapped even if Dirichlet boundary values that produce a bounce are imposed. To demonstrate this, we choose a boundary value inside the trapping region, close to the transition curve. A Bloch wall is launched from infinity towards the boundary. As the wall approaches the boundary, the simulation is stopped. The field configuration is saved and used as the initial condition for the next simulation run. In this new run we make a small change in the Dirichlet boundary value and move across the transition curve into the bouncing region. If the front core in the saved configuration is close enough to the boundary, the front will get trapped, even if Dirichlet boundary values that produce a bounce are imposed. An analytical explanation of coexistence for the FN model is given in the next section, which gives insights into the coexistence behavior in both models.

Summarizing, in both systems we have regions of trapped incoming Bloch fronts and bouncing Bloch fronts in the plane of boundary values. Critical slowing down of the front dynamics in proximity to a boundary is observed in both systems close to the transition curve. Also, the nontrivial stationary solutions in both systems remain stable across the transition curve, implying that they are not responsible for the critical behavior we see. Both systems exhibit the coexistence of bouncing and trapped solutions.

In the next section, we explain these numerical observations by deriving the mechanism that shows how $x$ and $c$ are coupled for Dirichlet boundary conditions in the FN model.

\section{ANALYSIS}

The goal of this section is to explain front interaction with boundaries, in terms of coupling of the evolution equations for the two degrees of freedom, front velocity $c$ and the front position $x$ in Eq. (7). It is shown that the coupling is the result of the spatial inhomogeneity sensed by the front as it approaches the boundary.

We restrict ourselves to the regime where $\epsilon / \delta$ is small and $\eta=\sqrt{\epsilon \delta}$ is finite. This restriction leads to a very sharp spatial variation of $u(x, t)$ field at the core of the front. The slowly varying $v(x, t)$ field can be considered constant in this core region. Hence the $v(x, t)$ field is represented by a single value $v_{f}$, at the point where the $u(x, t)$ field has zero value. We also restrict ourselves to small front velocities $c$, which can be done, by either making $\eta_{c}-\eta$ small or choosing a large $a_{1}$.

The restrictions described above lead to a drastic reduction in the number of degrees of freedom used to describe the front. As opposed to a front description based on the whole model Eq. (4), a sharp front (fast spatial variation of $u$ at the core) can be thought of as a point particle with a definite position and velocity. The slowly varying $v$ variable can be thought of as a field associated with this particle that allows it to sense the boundary. A small velocity has a few simple implications. An addition of two kinds of perturbations, one that changes $v_{f}$ (velocity) slightly and the other that produces a local distortion in the $v(x, t)$ field far from the front position, to a slow moving and uniformly translating front, is followed by the disappearance of the distortion and the relaxation of the front back to constant velocity. The time scale on which the distortion vanishes is much faster than the scale on which $v_{f}$ relaxes back to its original value. In essence, we have two time scales, the slower one associated with nonsteady front motion.

We now employ the restrictions mentioned above to obtain a reduced description of Eq. (4). We solve Eq. (4) with a Dirichlet boundary condition $v=v_{b}$ at the left boundary. On the right boundary, which is at infinity, we have $v=-q^{-2}$, and $q^{2}=a_{1}+1 / 2$. Following Ref. [12, we have the following system of equations,

$$
\dot{x}=-\frac{3}{\eta \sqrt{2}} v_{f},
$$

and

$$
\begin{array}{ll}
v_{t}+q^{2} v-v_{r r}=-\frac{3}{\eta \sqrt{2}} v(0, t) v_{r}+1 & r \leq 0 \\
v_{t}+q^{2} v-v_{r r}=-\frac{3}{\eta \sqrt{2}} v(0, t) v_{r}-1 & r \geq 0 \\
v(-x, t)=v_{b}, \quad v(\infty, t)=-q^{-2} . &
\end{array}
$$

Eq. (9) implies that the velocity is proportional to the value of the $v(x, t)$ field at the sharp interface formed by the $u(x, t)$ field. Eq. (10) represents the evolution of the $v(x, t)$ field in a frame of reference which moves with the 
front. The variable $r$ is the spatial coordinate from the front position and $r=-x$ is the distance of the boundary to the left of the front.

We solve Eq. (10) perturbatively. The starting point of the perturbative expansion is to find a stationary Ising wall solution. Therefore, setting the time derivatives in Eq. (10) to zero and looking for an Ising wall solution, we get,

$$
\begin{array}{ll}
v_{r r}-q^{2} v+1=0 & r \leq 0, \\
v_{r r}-q^{2} v-1=0 & r \geq 0,
\end{array}
$$

with $v\left(0_{+}\right)=v\left(0_{-}\right)=0$ and $v(\infty)=-q^{-2}$. This ensures that that the Ising wall has $v_{f}=0$. The solution to Eq. (11) is,

$$
\begin{aligned}
& v^{(0)}=-q^{-2}\left(e^{q r}-1\right) \quad r \leq 0, \\
& v^{(0)}=q^{-2}\left(e^{-q r}-1\right) \quad r \geq 0 .
\end{aligned}
$$

Hence for the Ising wall at $r=-x$, we have, $v^{(0)}(-x)=$ $\left(1-e^{-q x}\right) / q^{2}$.

We look for traveling Bloch wall solutions as a perturbation to this uniquely defined Ising wall. Since the Bloch walls have a Dirichlet boundary condition $v(-x)=v_{b}$, the perturbative correction to the Ising wall should have a boundary value $v_{c}=v_{b}-\left(1-e^{-q x}\right) / q^{2}$ which changes as the front moves.

Let $\bar{v}$ be the perturbation. Then,

$$
v=\bar{v}+v^{(0)} \text {. }
$$

$\bar{v}$ is expanded in powers of $c$, the small perturbation parameter. Since the front bifurcation is a pitchfork, $\eta$ is expanded in powers of $c^{2}$.

$$
\begin{aligned}
\bar{v}(r, t, T) & =\sum_{n=1}^{\infty} c^{(n)} v^{(n)}(r, t, T) \\
\eta & =\eta_{c}(x)-c^{2} \eta_{1}(x)+c^{4} \eta_{2}(x) .
\end{aligned}
$$

$T=c^{2} t$ is the slower time scale responsible for nonsteady front motion. The coefficients of powers of $c$ in the expansion of $\eta$ are functions of $x$ to incorporate the broken translational invariance. Using Eq. (12) and Eq. (13) in Eq. (10), one obtains

$$
v_{t}^{(n)}+q^{2} v^{(n)}-v_{r r}^{(n)}=-\rho^{(n)}, \quad n=1,2,3 . .,
$$

with

$$
\begin{aligned}
\rho^{(1)} & =\frac{3}{\sqrt{2} \eta_{c}} v_{\mid r=0}^{(1)} v_{r}^{(0)} \\
\rho^{(2)} & =\frac{3}{\sqrt{2} \eta_{c}}\left[v_{\mid r=0}^{(1)} v_{r}^{(1)}+v_{\mid r=0}^{(2)} v_{r}^{(0)}\right] \\
\rho^{(3)} & =v_{T}^{(1)}+\frac{3 \eta_{1}}{\sqrt{2} \eta_{c}^{2}} v_{\mid r=0}^{(1)} v_{r}^{(0)} \\
& +\frac{3}{\sqrt{2} \eta_{c}}\left[v_{\mid r=0}^{(1)} v_{r}^{(2)}+v_{\mid r=0}^{(2)} v_{r}^{(2)}+v_{\mid r=0}^{(3)} v_{r}^{(0)}\right]
\end{aligned}
$$

We use Green's functions to solve the system of equations above. The general solution, given that we have found an appropriate Green's function $G\left(r, t \mid r^{\prime}, t^{\prime}\right)$ is,

$$
\begin{aligned}
v^{(n)}(r, t) & =\int_{t_{i}}^{t} \int G\left(r, t \mid r^{\prime}, t^{\prime}\right) \rho^{n}\left(r^{\prime}, t^{\prime}\right) d t^{\prime} d r^{\prime} \\
& +\int_{t_{i}}^{t} \int_{s^{\prime}}\left[G\left(r, t \mid r^{\prime}, t^{\prime}\right) \frac{\partial^{\prime} v^{(n)}\left(r^{\prime}, t^{\prime}\right)}{\partial n}\right. \\
& \left.-\frac{\partial^{\prime} G\left(r, t \mid r^{\prime}, t^{\prime}\right)}{\partial n} v^{(n)}\left(r^{\prime}, t^{\prime}\right)\right] d t^{\prime} d s^{\prime} \\
& +\int G\left(r, t \mid r^{\prime}, t_{i}\right) v^{(n)}\left(r^{\prime}, t_{i}\right) d r^{\prime} .
\end{aligned}
$$

The last term can be made zero by choosing an appropriate initial condition. The first term gives the influence of sources on the evolution of the $v(x, t)$ field. The second term incorporates the influence of boundary conditions. To apply Dirichlet boundary conditions one finds a Green's function which is zero at the left boundary. Since we have a semi-infinite domain, we use the method of images to write down the Green's function

$$
\begin{aligned}
& G\left(r, t \mid r^{\prime}, t^{\prime}\right)=\frac{e^{-q^{2}\left(t-t^{\prime}\right)}}{\sqrt{4 \pi\left(t-t^{\prime}\right)}} \exp \left[-\frac{\left(r-r^{\prime}\right)^{2}}{4\left(t-t^{\prime}\right)}\right] \\
& -\frac{e^{-q^{2}\left(t-t^{\prime}\right)}}{\sqrt{4 \pi\left(t-t^{\prime}\right)}} \exp \left[-\frac{\left(r+r^{\prime}+2 x\left(t^{\prime}\right)\right)^{2}}{4\left(t-t^{\prime}\right)}\right],
\end{aligned}
$$

where the second term is the image of the first and $G=0$ at $r=-x$, the boundary. Proceeding with the calculation of source effects in Eq. (16), we have,

$$
\begin{aligned}
& v^{(n)}(r, t)=\int_{-x\left(t^{\prime}\right)}^{\infty} \int_{0}^{t} \frac{e^{-q^{2}\left(t-t^{\prime}\right)}}{\sqrt{4 \pi\left(t-t^{\prime}\right)}}\left\{\exp \left[-\frac{\left(r-r^{\prime}\right)^{2}}{4\left(t-t^{\prime}\right)}\right]\right. \\
& \left.-\exp \left[-\frac{\left(r+r^{\prime}+2 x\left(t^{\prime}\right)\right)^{2}}{4\left(t-t^{\prime}\right)}\right]\right\} \rho^{(n)}\left(r^{\prime}, t^{\prime}\right) d t^{\prime} d r^{\prime} .
\end{aligned}
$$

The Green's function terms in Eq. (18) above contain exponentials of functions of time $t^{\prime}$ that possess a maxima at some time $t_{o}^{\prime}$. Hence most of the contribution to the integral comes around this maximum value, which is approximately given by $t-t_{o}^{\prime}=\left|r-r^{\prime}\right| / 2 q$ for the first integral, and $t-t_{o}^{\prime}=\left|r+r^{\prime}+2 x\left(t_{o}^{\prime}\right)\right| / 2 q$ for the second. If the width of the maxima peak is less than these time differences, we can take the limit $t \rightarrow \infty$ in the integrals above. Further, if all the source terms are smoothly varying functions of $t^{\prime}$, one could perform a steepest descent approximation to the integrals above assuming that the maxima peak is nearly a gaussian and sharp enough. Physically, as a consequence of both reaction and diffusion, the configuration of fields at present time $t$ in Eq. (14) is determined by the time behavior of sources in an earlier small time window, in which reaction and diffusion mechanisms combine to produce the maximum rate of change of the $v(x, t)$ field. At all other times, either reaction or diffusion is individually dominant and 
not able to produce a combined high rate of change of $v(x, t)$. This is unlike pure diffusion, where all the time history of sources is required to give the field configuration at present time. Performing the steepest descent calculation, the time portion of the integral in Eq. (18) can be eliminated and it reduces to,

$v^{(n)}(r)=\int_{-x}^{\infty}\left[g+\frac{p e^{-\left(r+r^{\prime}+2 x\right) f}}{\sqrt{2} \sqrt{a\left(r+r^{\prime}+2 x\right)+b}}\right] \rho^{(n)}\left(r^{\prime}\right) d r^{\prime}$,

where,

$$
\begin{aligned}
g & =e^{-q\left|r-r^{\prime}\right|} / 2 q, \\
p & =\left(\dot{x} \pm \sqrt{\dot{x}^{2}+q^{2}}\right) / 2 q^{2}, \\
f & =q^{2} p+1 / 4 p, \\
a & =\ddot{x} p^{2} \\
b & =2 \dot{x}^{2} p^{2}+2 \dot{x} p+1 / 2 .
\end{aligned}
$$

The first factor in the bracelets $g$ dominates the evolution far away from the boundary, while the second factor is responsible for sensing the boundary. One notices that $\dot{x}$ and $\ddot{x}$, the velocity and acceleration of the front are involved in the reduced Green's functions in Eq. (19). If $\dot{x}$ and $\ddot{x}$ are neglected in the expressions above (justifiably so since we are close to the front bifurcation), solving Eq. (14) reduces to solving

$$
q^{2} v^{(n)}-v_{r r}^{(n)}+\rho^{(n)}=0, \quad n=1,2,3 . .
$$

This is what the authors do in Ref. 12], although no boundary influence is considered. Solving Eq. (19) further for $n=1,2,3$, and requiring the smoothness of the front at all orders, we get the equation for the evolution of $v_{f}=c v^{(1)}$.

$$
\begin{aligned}
\dot{v}_{f} & =-\frac{v_{f}}{\sigma}\left[\frac{\partial \sigma}{\partial x} \frac{\partial x}{\partial t}+\frac{\partial \sigma}{\partial \dot{x}} \frac{\partial \dot{x}}{\partial t}+\frac{\partial \sigma}{\partial \ddot{x}} \frac{\partial \ddot{x}}{\partial t}\right] \\
& +\frac{4 q^{2} \sigma \sqrt{2}}{9}\left(\eta_{c}-\eta\right) v_{f}-\frac{\sigma^{2}}{6} v_{f}^{3}
\end{aligned}
$$

where,

$$
\begin{aligned}
\sigma & =3 / \sqrt{2} \eta_{c} \\
& =\left[\left(2-e^{-2 q x}\right) / 4 q^{3}+S_{1}+S_{2}\right]^{-1},
\end{aligned}
$$

and

$$
\begin{gathered}
S_{1}=\int_{-x}^{0} \frac{p e^{-\left(r+r^{\prime}+2 x\right) f}}{\sqrt{2} \sqrt{a\left(r+r^{\prime}+2 x\right)+b}}\left[\frac{-e^{q r^{\prime}}}{q}\right] d r^{\prime}, \\
S_{2}=\int_{0}^{\infty} \frac{p e^{-\left(r+r^{\prime}+2 x\right) f}}{\sqrt{2} \sqrt{a\left(r+r^{\prime}+2 x\right)+b}}\left[\frac{-e^{-q r^{\prime}}}{q}\right] d r^{\prime} .
\end{gathered}
$$

Hence $S_{1}, S_{2}$ and so $\sigma$ depend on $x, \dot{x}, \ddot{x}$. Neglecting $\dot{x}$ and $\ddot{x}$ in $S_{1}$ and $S_{2}, \sigma$ reduces to,

$$
\sigma=\frac{4 q^{3}}{2+(1+2 q x) e^{-2 q x}} .
$$

Far away from the boundary influence, one recovers $\sigma=2 q^{3}$ as expected. Therefore, one of the effects of proximity to the boundary is spatial dependence of the critical bifurcation parameter $\eta_{c}$. Due to this form of $\sigma$, where its dependence on velocity and acceleration is ignored and its spatial derivative falls off sharply with $x$, all its derivatives in Eq. (21) can be neglected. Thus we have,

$$
\dot{v}_{f}=\frac{4 q^{2} \sigma \sqrt{2}}{9}\left(\eta_{c}-\eta\right) v_{f}-\frac{\sigma^{2}}{6} v_{f}^{3} .
$$

We now derive the effect of boundary conditions on the evolution of the front. Taking the derivative of $G$ with respect to $r^{\prime}$ in Eq. (17) and substituting it in Eq. (16), the boundary contribution is found to be

$$
\begin{aligned}
\phi_{1} & =-\int_{0}^{t} \frac{v_{c}\left[x\left(t^{\prime}\right)\right] e^{-q^{2}\left(t-t^{\prime}\right)}}{2 \pi^{1 / 2}\left(t-t^{\prime}\right)^{3 / 2}} \\
& \times\left[r+x\left(t^{\prime}\right)\right] \exp \left[-\frac{\left[r+x\left(t^{\prime}\right)\right]^{2}}{4\left(t-t^{\prime}\right)}\right] d t^{\prime} .
\end{aligned}
$$

Now we are only interested in the contribution of the boundary terms at the front position, $\left.\phi_{1}\right|_{r=0}$. This extra term gets added on to $v_{f}$, the value of the $v$ field at the front position, thus incorporating the influence of the specific Dirichlet boundary condition $v_{b}$ on the front velocity. From now on $\phi_{1}$ will stand for $\left.\phi_{1}\right|_{r=0}$.

Nonsteady front motion represented by $\dot{v}_{f}$ involves time derivatives, hence the time derivative of $\phi_{1}$ gives the influence of the boundary condition in accelerating fronts,

$$
\begin{aligned}
\phi_{2} & =\frac{\partial \phi_{1}}{\partial t} \\
& =-\int_{0}^{t} \frac{v_{c}\left[x\left(t^{\prime}\right)\right] e^{-q^{2}\left(t-t^{\prime}\right)}}{2 \pi^{1 / 2}\left(t-t^{\prime}\right)^{3 / 2}} \exp \left[-\frac{\left[x\left(t^{\prime}\right)\right]^{2}}{4\left(t-t^{\prime}\right)}\right] \\
& \times\left(q^{2} x\left(t^{\prime}\right)+\frac{3 x\left(t^{\prime}\right)}{\left(t-t^{\prime}\right)}-\frac{\left[x\left(t^{\prime}\right)\right]^{3}}{4\left(t-t^{\prime}\right)^{2}}\right) d t^{\prime} .
\end{aligned}
$$

Incorporating these boundary effects in Eq. (23), $v_{f} \rightarrow$ $v_{f}+\phi_{1}$, and $\dot{v}_{f} \rightarrow \dot{v}_{f}+\phi_{2}$, we get

$$
\dot{v_{f}}=\phi_{2}+\frac{4 q^{2} \sigma \sqrt{2}}{9}\left(\eta_{c}-\eta\right)\left(v_{f}+\phi_{1}\right)-\frac{\sigma^{2}}{6}\left(v_{f}+\phi_{1}\right)^{3} .
$$

Eq. (9) and Eq. (26) constitute the coupling of the two degrees of freedom $c$ and $x$ or equivalently $v_{f}$ and $x$ in the presence of a spatial inhomogeneity introduced by Dirichlet boundary conditions.

One of the stationary points of the system above is $\left(x, v_{f}\right)=\left(-\ln \left(1-v_{b} q^{2}\right) / q, 0\right)$. This is the point closely connected with the dynamics we now describe. We look at the parameter $v_{b}$ in three different regimes, $v_{b}<0$, $0<v_{b}<1 / q^{2}$, and $v_{b}>1 / q^{2}$. The region of physical 


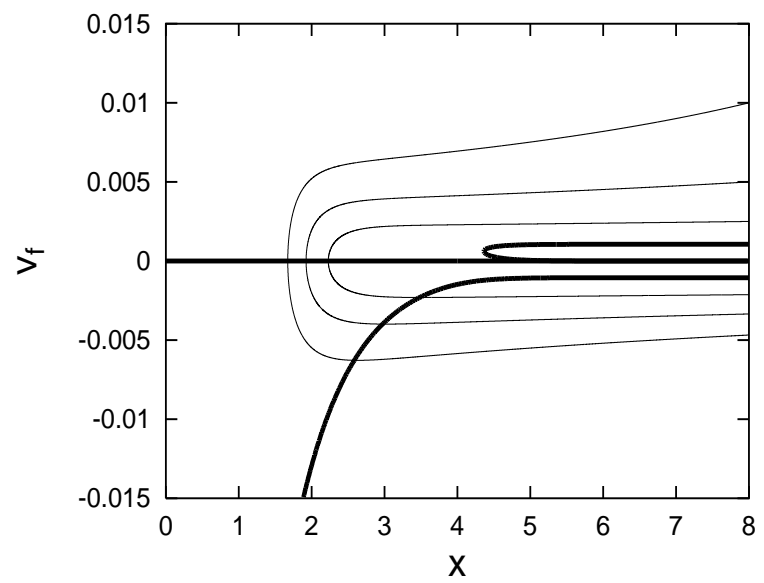

FIG. 5: The dark curves are the nullclines, grey curves are the solutions to Eq. (26) with different initial conditions. These are the typical flows in the regime $v_{b}<0$, where incoming fronts always bounce. There are no fixed points and $v_{b}=$ $-0.1, a 1=9.9, \epsilon=.001, \delta=1.0$.

interest is $x>0 . v_{f}>0$ implies a front moving towards the boundary and $v_{f}<0$ is a front moving away.

Although, the convolution integrals $\phi_{1}$ and $\phi_{2}$ represent the complete influence of boundary conditions, we wish to approximate them to obtain a simpler picture that preserves the qualitative features of the complete integrals. The integrals involve exponentials of functions that have a maxima at time $t_{o}^{\prime}$, and hence most of the contribution is around this maxima. For small front velocities this maxima is given by $t-t_{o}^{\prime}=x / 2 q$. The width of this maxima peak is given by $(x / 2 q)^{\frac{3}{2}}$. If $x / 2 q>>$ $(x / 2 q)^{\frac{3}{2}}$, which it is for a very sharp peak, a steepest descent approximation can be made. A greater inequality implies a better approximation. The approximation gives, $\phi_{1}=-v_{c}(x) e^{-q x}$ and $\phi_{2}=\partial \phi_{1} / \partial t=-3 \phi_{1} q / x$, where it is again assumed that the velocities are small.

In the regime $v_{b}<0$ the fixed point is in the negative $x$ region and is an unstable spiral. The term involving $1 / x$ in the expression for $\phi_{2}$ prevents flows from crossing the negative $x$ region to the positive one and vice versa. Therefore the fixed point does not influence the $x>0$ flows. Figure 5 shows the nullclines and the typical flows. The grey flow curves show the turning around of fronts at the boundary. It is notable that the nullclines are not followed well by the flow curves and cannot predict the dynamics of front reversal. Generally, the flows will agree better with the nullclines when the relaxation rate, determined by $\eta_{c}-\eta$, is larger, although the jump from one nullcline to another can only be explained by the dynamical equations.

As one increases $v_{b}$ and enters the $0<v_{b}<1 / q^{2}$ regime, the fixed point crosses over into the $x>0$ region. The fixed point now influences the flows close to the boundary. Instead of being an unstable spiral it now is a saddle with two distinct real eigenvalues, giving rise

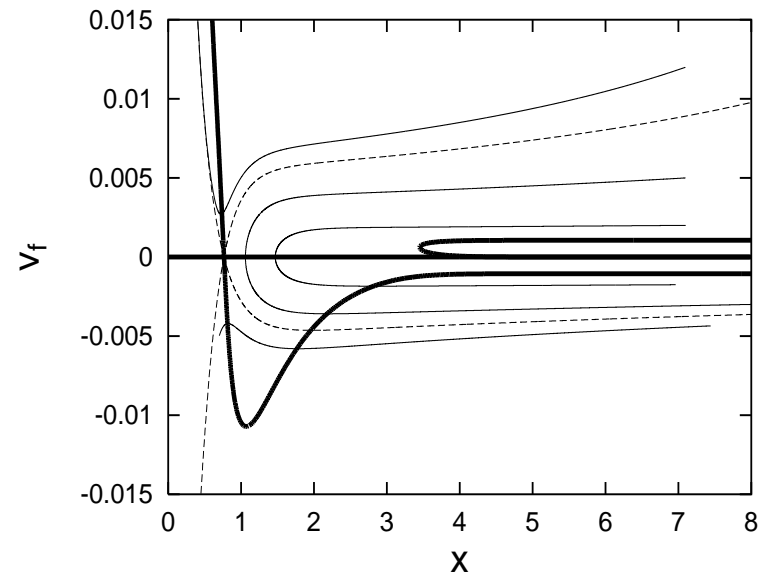

FIG. 6: Flows in the coexistence region $0<v_{b}<1 / q^{2}$. Dark curves are the nullclines, which intersect at the fixed point. Grey curves are the solutions to Eq. (26), with different initial conditions. The dashed lines represent the invariant manifolds separating basins of attraction and bouncing. $v_{b}=0.088$, $a 1=9.9, \epsilon=.001, \delta=1.0$.

to stable and unstable manifolds. The eigenvalues are

$$
\lambda_{ \pm}=\frac{\lambda_{o} \pm \sqrt{\lambda_{o}^{2}+4 \lambda_{x}}}{2}
$$

where $\lambda_{o}=4 q^{2} \sigma \sqrt{2} / 9\left(\eta_{c}-\eta\right)$, and

$$
\lambda_{x}=-\frac{\left(1-v_{b} q^{2}\right)}{\sqrt{2} \eta}\left[\frac{9 q}{\ln \left(1-v_{b} q^{2}\right)}\right] .
$$

As $v_{b} \rightarrow 1 / q^{2}$, the fixed point moves away from the boundary towards positive infinity and $\lambda_{+} \rightarrow \lambda_{o}$, which is the eigenvalue for an unstable Ising wall far from the boundary influence. Also, in the same limit, $\lambda_{-} \rightarrow 0$, where the zero eigenvalue is associated with spatial homogeneity (translational invariance). This explains the critical slowing down observed in the last section. Trajectories wandering close to this fixed point near criticality $\left(v_{b} \rightarrow 1 / q^{2}\right)$ will rebound or trap on a slower time scale, compared to a relatively faster dynamics when the fixed point is further away from criticality.

The time scales close to the fixed point are controlled by $\lambda$, which has two constituents, $\lambda_{o}$ and $\lambda_{x} . \lambda_{o}$ is associated with the slow time scale $T=c^{2} t$, which depends on the distance to the front bifurcation $\eta_{c}-\eta$, and can be made arbitrarily small. Therefore, close to the front bifurcation, $\lambda_{o}$ can be neglected in Eq. (27) and the eigenvalues reduce to $\left|\lambda_{ \pm}\right|=\sqrt{\lambda_{x}}$. This is the new time scale determined solely by the influence of boundary conditions and is the dominant time scale in the nonadiabatic limit of extremely slow velocities. Bloch wall trajectories close to the saddle, which either trap or bounce, evolve on this time scale.

Typical flows are plotted in Fig. [6 The triangle shows the fixed point. The dashed lines are the invariant sets, 


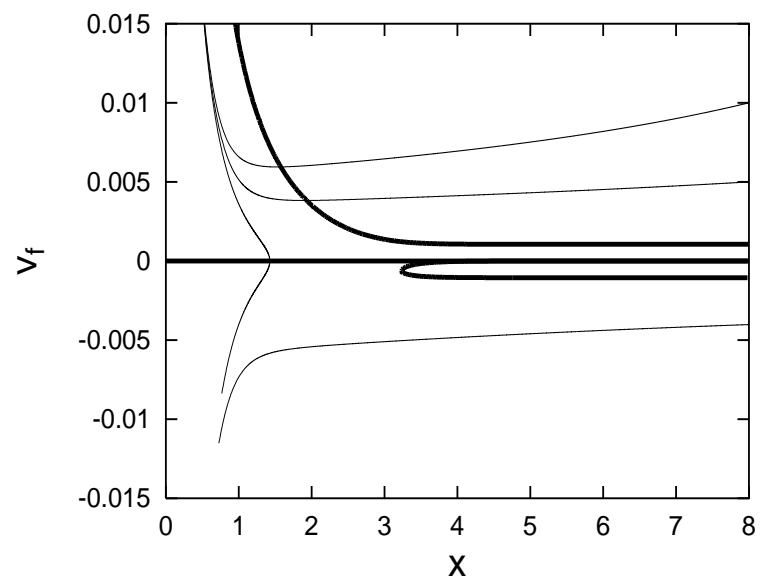

FIG. 7: Flows in the regime $v_{b}>1 / q^{2}$. Again there are no fixed points present and all the flows get trapped at the boundary. $v_{b}=0.1, a 1=9.9, \epsilon=.001, \delta=1.0$

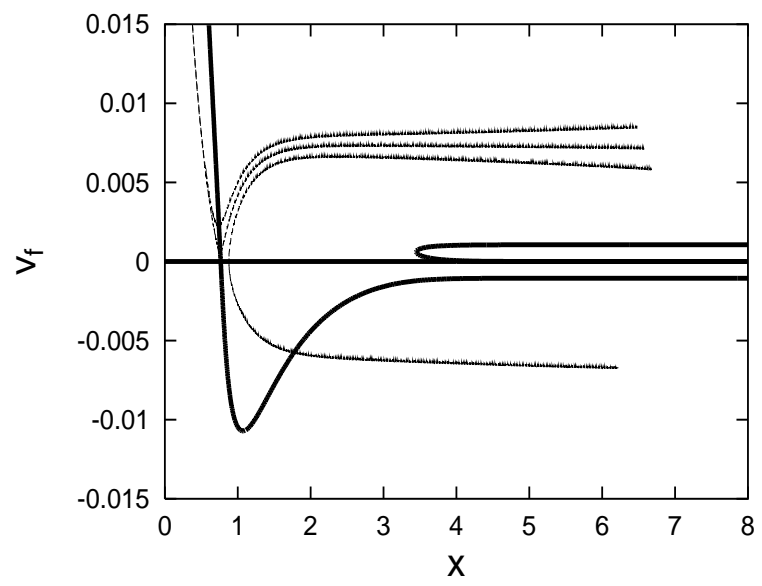

FIG. 8: This shows the actual simulation of Eq. (4) for $v_{b}=$ $0.088, a 1=9.9, \epsilon=.001, \delta=1.0$ The thick dark lines are the nullclines. The thin lines are the trajectories.

with arrows showing the direction of flow. The invariant sets separate basins of attraction of flows towards the boundary and basins of reflection away from it. This explains the coexistence region. If the initial velocity and position of the front is inside the attraction basin, it gets trapped at the boundary, if not, it rebounds. The numerically obtained transition curve in the last section is a manifestation of this behavior, where initial conditions are fixed and varying the boundary values leads to a crossover of the initial condition from a basin of attraction to that of repulsion. It should be noted that the coexistence region can only be explained by the presence of the fixed point and the dynamics associated with it. An adiabatic analysis relying on nullclines is not the complete picture.

For $v_{b}>1 / q^{2}$ the fixed point no longer exists. Figure 7 shows the flows in this regime. Incoming fronts always get trapped. Since no fixed points are present, the flow qualitatively does what the nullclines do, as is the case in the $v_{b}<0$ regime.

Summarizing, transition from bouncing to trapped fronts is governed by a fixed point close to the boundary. This fixed point gives rise to the coexistence behavior, and is absent in regimes where only trapping or bouncing occurs. We conclude our analysis by pointing out that solutions of Eq. (23), with approximated $\phi_{1}$ and $\phi_{2}$, agree well qualitatively with the solution of the FN model Eq. (4) plotted in Fig. 8

\section{CONCLUSION}

We have studied Bloch front motion in the CGL and FN models in the presence of spatial inhomogeneity introduced by Dirichlet boundary conditions at the boundary. We have shown similar features in the behavior of Bloch fronts close to the boundary in both systems. There is a transition from trapping (annihilation) to bouncing of incoming Bloch fronts for both systems as a function of Dirichlet boundary values. Also for certain boundary values trapped and bouncing Bloch fronts coexist.

In the sharp front and slow velocity regime for the FN model, we were able to give a mathematical mechanism for the trapping, bounce and coexistence of the two. This involves the coupling of the front velocity $c$ and front position $x$ close to the boundary. Essentially, Dirichlet boundary conditions act as a barrier to Bloch fronts coming in. It is shown that this barrier may or may not be penetrated depending upon how fast or far away the incoming Bloch wall is created. It is never penetrated, if $v_{b}<0$. It is always penetrated if $v_{b}>1 / q^{2}$.

One could also derive how $c$ and $x$ are coupled close to a boundary for zero flux boundary conditions, by using a modified Green's function, with a zero derivative at the boundary. Zero flux boundary conditions [4] show rich behavior too, (breathing fronts) and the dynamics can be explained by such a calculation.
[1] K. J. Lee, W .D. McCormick, Q. Ouyang, and H. L. Swinney, Science 261, 192 (1993).

[2] K. J. Lee, W. D. McCormick, J. E. Pearson, and H. L. Swinney, Nature 369 , 215 (1994).

[3] K. J. Lee and H. L. Swinney, Phys. Rev. E. 51 , 1899 (1995).
[4] D. Haim, G. Li, Q. Ouyang, W. D. McCormick, H. L. Swinney, A. Hagberg, and E. Meron, Phys. Rev. Lett. 77, 190 (1996).

[5] G. Haas, M. Bar, I. G. Kevrekidis, P. B. Rasmussen, H.H. Rotermund, and G. Ertl, Phys. Rev. Lett. 75, 3560 (1995). 
[6] G. Li, Q. Ouyang, and H. L. Swinney, J. Chem. Phys. 105, 10830 (1996).

[7] P. Coullet, J. Lega, B. Houchmanzadeh, and J. Lajzerowicz, Phys. Rev. Lett. 65, 1352 (1990).

[8] T. Frisch, S. Rica, P. Coullet, and J. M. Gilli, Phys. Rev. Lett. 72, 1471 (1994).

[9] S. Nasuno, N. Yoshimo, and S. Kai, Phys. Rev. E. 51, 1598 (1995).

[10] A. Hagberg and E. Meron, Phys. Rev. Lett. 72, 2494 (1994).

[11] C. Elphick, A. Hagberg, and E. Meron, Phys. Rev. E. 51, 3052 (1995).

[12] A. Hagberg, E. Meron, I. Rubinstein, and B. Zaltzman, Phys. Rev. Lett. 76, 427 (1996).

[13] A. Hagberg and E. Meron, Chaos 4, 477 (1994).

[14] V. Castets, E. Dulos, J. Boissonade, and P. De Kepper, Phys. Rev. Lett. 64, 2953 (1990).

[15] Q. Ouyang and H. L. Swinney, Chaos 1, 411 (1991).

[16] I. Lengyel, S. Kadar, and I. R. Epstein, Phys. Rev. Lett. 69, 2729 (1992).

[17] W. van Saarloos, "The Complex Ginzburg Landau equa- tion for Beginners", in : Proceedings of the Santa Fe workshop on 'Spatio-Temporal Patterns in Nonequilibrium Complex Systems', P. E. Cladis and P. PalffyMuhoray, eds.,p19-31 (Addison Wesley, Chicago, 1994).

[18] C. Elphick, A. Hagberg, B. A. Malomed, and E. Meron, Phys. Lett. A 230(1-2) 33-37 (1997).

[19] W. van Saarloos, Phys. Rev. Lett. 58, 2571 (1987)

[20] W. van Saarloos, Phys. Rev. A. 39, 6367 (1989)

[21] A. Hagberg and E. Meron, Nonlinearity 7, 805 (1994).

[22] L. Kuhnert and H. J. Krug, J. Phys. Chem. 91, 730 (1987).

[23] L. Kuhnert, H. J. Krug and L. Pohlman, J. Phys. Chem. 89, 2022 (1985)

[24] Zs. Nagy-Ungvarai, J. J. Tyson and B. Hess, J. Phys. Chem. 93, 707 (1989).

[25] Zs. Nagy-Ungvarai, S. C. Muller and J. J. Tyson, J. Phys. Chem. 93, 2760 (1989).

[26] P. M. Woods and J. Ross, J. Chem. Phys. 82, 1924 (1985). 